\title{
Holographic Entanglement of Purification from Conformal Field Theories
}

\author{
Pawel Caputa, ${ }^{1}$ Masamichi Miyaji, ${ }^{1}$ Tadashi Takayanagi, ${ }^{1,2}$ and Koji Umemoto ${ }^{1}$ \\ ${ }^{1}$ Center for Gravitational Physics, Yukawa Institute for Theoretical Physics, Kyoto University, \\ Kitashirakawa Oiwakecho, Sakyo-ku, Kyoto 606-8502, Japan \\ ${ }^{2}$ Kavli Institute for the Physics and Mathematics of the Universe (WPI), University of Tokyo, Kashiwa, Chiba 277-8582, Japan
}

(Received 21 December 2018; published 21 March 2019)

\begin{abstract}
We explore a conformal field theoretic interpretation of the holographic entanglement of purification, which is defined as the minimal area of the entanglement wedge cross section. We argue that, in $\mathrm{AdS}_{3} / \mathrm{CFT}_{2}$, the holographic entanglement of purification agrees with the entanglement entropy for a purified state, obtained from a special Weyl transformation, called path-integral optimizations. By definition, this special purified state has minimal path-integral complexity. We confirm this claim in several examples.
\end{abstract}

DOI: $10.1103 /$ PhysRevLett.122.111601

Introduction.-Quantum entanglement provides us a key to understanding how gravity emerges from field theories. This is manifest in the anti-de Sitter space (AdS)/conformal field theory (CFT) correspondence (or holography) [1]. In AdS/CFT, the entanglement entropy, the unique measure of quantum entanglement for pure states, is computed as a minimal area in AdS $[2,3]$.

For mixed states, entanglement entropy can measure neither quantum entanglement nor correlations between two subsystems. Recently, it was conjectured that a quantity called entanglement of purification [4], which is a good measure of total correlations, has a simple geometric interpretation in AdS/CFT, called the holographic entanglement of purification [5,6]. This quantity is a natural extension of entanglement entropy for mixed states. An extension to multipartite correlations is also given in Ref. [7].

The entanglement of purification (EOP), written as $E_{P}\left(\rho_{A B}\right)$, is a measure of correlations between two subsystems $A$ and $B$ for a mixed state $\rho_{A B}$ and is defined as follows [4]. Consider a purification of $\rho_{A B}$, given by $|\Psi\rangle_{A \tilde{A} B \tilde{B}}$, by enlarging the Hilbert space as $\mathcal{H}_{A} \otimes \mathcal{H}_{B} \rightarrow$ $\mathcal{H}_{A} \otimes \mathcal{H}_{B} \otimes \mathcal{H}_{\tilde{A}} \otimes \mathcal{H}_{\tilde{B}}$ such that

$$
\rho_{A B}=\operatorname{Tr}_{\tilde{A} \tilde{B}}\left[|\Psi\rangle_{A \tilde{A} B \tilde{B}}\left\langle\left.\Psi\right|_{A \tilde{A} B \tilde{B}}\right] .\right.
$$

Among infinitely many different choices of the purifications $|\Psi\rangle_{A \tilde{A} B \tilde{B}}$, the EOP is defined by minimizing the entanglement entropy $S_{A \tilde{A}}=-\operatorname{Tr}\left[\rho_{A \tilde{A}} \log \rho_{A \tilde{A}}\right]$ as

Published by the American Physical Society under the terms of the Creative Commons Attribution 4.0 International license. Further distribution of this work must maintain attribution to the author(s) and the published article's title, journal citation, and DOI. Funded by SCOAP ${ }^{3}$.

$$
E_{P}\left(\rho_{A B}\right)=\min _{|\Psi\rangle_{A \tilde{B} B \tilde{B}}}\left[S_{A \tilde{A}}\right]
$$

The holographic entanglement of purification (HEOP) $E_{W}\left(\rho_{A B}\right)$ is defined by the area of the minimal cross section of entanglement wedge, denoted by $\Sigma_{A B}^{\min }$ :

$$
E_{W}\left(\rho_{A B}\right)=\frac{A\left(\Sigma_{A B}^{\min }\right)}{4 G_{N}},
$$

and the equality $E_{W}\left(\rho_{A B}\right)=E_{P}\left(\rho_{A B}\right)$ was conjectured in Refs. [5,6] based on quantum information theoretic properties. In this Letter, we focus on static backgrounds and always take a canonical time slice in the AdS.

The entanglement wedge is the region inside the AdS which is dual to the density matrix $\rho_{A B}[8-10]$. Therefore, the HEOP [Eq. (3)] is quite fundamental to understanding how the geometry in gravity corresponds to density matrices in CFTs. It also has an important meaning in the bit threads interpretation of AdS/CFT [11-14].

Nevertheless, direct comparisons between the HEOP and EOP have not been done so far, mainly because the minimization procedure in Eq. (2) is very hard in quantum field theories. Refer to Refs. $[6,15,16]$ for numerical lattice calculations of EOP in free field theories. Also in Refs. [17-19], connections between $E_{W}\left(\rho_{A B}\right)$ and quantities other than the EOP have been proposed. For other recent progresses on the HEOP, refer to Refs. [20-32]

In this Letter, we would like to present a direct comparison between the HEOP $E_{W}\left(\rho_{A B}\right)$ and the entanglement entropy $S_{A \tilde{A}}$ for a special class of purification $|\Psi\rangle_{A \tilde{A} B \tilde{B}}$, obtained by the path-integral optimization [33-35]. We will focus on several examples in $\mathrm{AdS}_{3} / \mathrm{CFT}_{2}$ and will find both quantities always agree with each other in the regime of validity of our computations. 
Path-integral optimization. - Consider a two-dimensional (2D) CFT on a Euclidean flat space $R^{2}$, which is described by the complex coordinate $(w, \bar{w})=(\xi+i \tau, \xi-i \tau)$ with the metric

$$
d s^{2}=d w d \bar{w}=d \tau^{2}+d \xi^{2} .
$$

The theory is invariant under the Weyl transformation of the metric:

$$
d s^{2}=e^{2 \phi(\tau, \xi)}\left(d \tau^{2}+d \xi^{2}\right),
$$

where $\phi(\tau, \xi)$ is any function.

The path-integral optimization [33] is a special choice of Weyl transformation which (i) preserves the quantum state $|\Psi\rangle$ at a particular time $\tau=\tau_{0}$ and (ii) minimizes the pathintegral complexity $C_{L}[\phi]$ (defined below).

Since the wave functional of $|\Psi\rangle$ can be computed by the Euclidean path integral on the lower half plane $-\infty<\tau \leq \tau_{0}$, the first condition (i) is given by the boundary condition

$$
e^{2 \phi\left(\tau_{0}, \xi\right)}=1 .
$$

The path-integral complexity is defined by the Liouville action

$$
C_{L}[\phi]=\frac{c}{24 \pi} \int_{-\infty}^{\tau_{0}} d \tau \int_{-\infty}^{\infty} d \xi\left(\left(\partial_{\tau} \phi\right)^{2}+\left(\partial_{\xi} \phi\right)^{2}+\frac{e^{2 \phi}}{\epsilon^{2}}\right),
$$

where $\epsilon$ is the UV cutoff or equally lattice spacing and $c$ is the central charge. The reason why we identify $C_{L}[\phi]$ with the complexity in path integrations is because the path-integral measure is proportional to $e^{C_{L}[\phi]}$ due to the conformal anomaly [36]. This quantity $C_{L}[\phi]$ provides a field theoretic counterpart of holographic complexity [37] as explained in Ref. [38].

In this argument, we consider the discretization of the path integral such that each cell has the area $\epsilon^{2}$. The original flat metric [Eq. (4)] corresponds to a square lattice with lattice spacing $\Delta \xi=\Delta \tau=\epsilon$. The optimization which changes the metric such that $e^{2 \phi} \leq 1$ means coarse-graining lattice sites such that $\Delta \xi=\Delta \tau=\epsilon e^{-\phi}$.

The minimization of $C_{L}[\phi]$ can be found by solving the Liouville equation $\left(\partial_{\tau}^{2}+\partial_{\xi}^{2}\right) \phi=e^{2 \phi} / \epsilon^{2}$. Choosing $\tau_{0}=-\epsilon$ using the time translational symmetry, we find the solution which satisfies the condition [Eq. (6)]

$$
e^{2 \phi}=\frac{\epsilon^{2}}{\tau^{2}}
$$

This describes a hyperbolic space $\mathrm{H}_{2}$. Note that in Ref. [33] we had $e^{2 \phi}=1 / z^{2}$ because we defined $z=-\epsilon$ with the rescaling $e^{\phi} \rightarrow \epsilon e^{\phi}$. This is the simplest example of the path-integral optimization. Even though we optimized a pure state $|\Psi\rangle$, it is straightforward to extend the formulation such that we optimize a given mixed state density matrix.

Optimization of single interval.-Consider the case where the subsystem $A B(\equiv A \cup B)$ is given by a single interval in a vacuum. We parametrize the subsystem $A$ and $B$ as follows:

$A=[a, p], \quad B=[p, b], \quad(-\infty<a<p<b<\infty)$,

where they share the point $P$ given by $y=p$. The reduced density matrix $\rho_{A B}$ is given by the Euclidean path integral over a complex plane with a slit along $A B$, whose coordinate is denoted by $y$. Next we consider a purification $|\Psi\rangle_{A \tilde{A} B \tilde{B}}$ of $\rho_{A B}$ by introducing the subsystem $\tilde{A}$ and $\tilde{B}$ as in Fig. 1 such that they share the point $Q$ parametrized by $y=q$.

The original definition of EOP is the minimum of $S_{A \tilde{A}}$ against any purifications [Eq. (2)]. Here we would like to restrict ourselves to a class of purification which is realized by the Weyl transformation [Eq. (5)] and focus on the one given by the path-integral optimization (i.e., the one which minimizes $C_{L}[\phi]$ ). The purification condition [Eq. (1)] is equivalent to the requirement [Eq. (6)].

To find the optimization, let us map the $y$ space into an upper half plane ( $w$ plane) by

$$
w=\sqrt{\frac{y-a}{b-y}} .
$$

The points $P$ and $Q$ are mapped into $w_{P}=$ $-\sqrt{(p-a / b-p)}$ and $w_{Q}=i \sqrt{(q-a / q-b)}$. Note that such a conformal map transforms a solution of the Liouville equation in one coordinate into that in another coordinate. In the above, we mapped into the $w$ plane as we know very well solutions of the Liouville equation in the setup of $w$ coordinate.

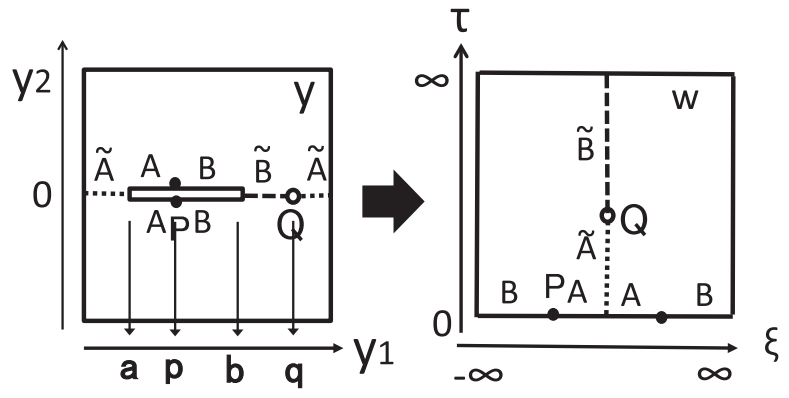

FIG. 1. Conformal transformation between a complex plane with one slit and an upper half plane. 
Since the path-integral optimization on the upper half plane is given by the hyperbolic space [Eq. (8)], the final optimized metric reads

$$
d s^{2}=\frac{\epsilon^{2}}{\tau^{2}} d w d \bar{w}=\frac{\epsilon^{2}}{\tau^{2}} \frac{(b-a)^{2}}{4|b-y|^{3}|y-a|} d y d \bar{y} \equiv e^{2 \tilde{\phi}} d y d \bar{y} .
$$

The coordinate $\tau$ takes values in the range $-\infty<\tau<-\delta_{1}$, where $\delta_{1}(>0)$ is an appropriate cutoff so that we maintain the condition Eq. (1). However, a straightforward analysis shows the detail of this regularization is not important in our calculations. In this setup, we have $e^{2 \tilde{\phi}_{P}}=1$, which follows from the condition Eq. (4), and we find $e^{2 \tilde{\phi}_{Q}}=\left[\epsilon^{2}(b-a)^{2} / 4(q-a)^{2}(q-b)^{2}\right]$, where $\tilde{\phi}_{P}$ and $\tilde{\phi}_{Q}$ are the value of $\tilde{\phi}$ at $P$ and $Q$.

By identifying the purification $|\Psi\rangle_{A \tilde{A} B \tilde{B}}$ in Eq. (1) with the path integration on the upper half plane in the $y$ coordinate with the optimized metric [Eq. (11)], the entanglement entropy $S_{A \tilde{A}}=S_{B \tilde{B}}$ is found to be

$$
\begin{aligned}
S_{A \tilde{A}} & =\frac{c}{3} \log \left(\frac{q-p}{\epsilon}\right)+\frac{c}{6} \tilde{\phi}_{P}+\frac{c}{6} \tilde{\phi}_{Q} \\
& =\frac{c}{6} \log \left[\frac{(b-a)(q-p)^{2}}{2 \epsilon(q-a)(q-b)}\right],
\end{aligned}
$$

where in the first line we performed a scale transformation of the standard formula of Ref. [39]. Remember that the entanglement entropy can be found from the two point function of twist operators in the replica method and this transforms in a standard way under the Weyl transformation. Then we minimize $S_{A \tilde{A}}$ with respect to $q$, leading to

$$
\begin{gathered}
S_{A \tilde{A}}^{\min }=\frac{c}{6} \log \left[\frac{2(p-a)(b-p)}{\epsilon(b-a)}\right], \\
\text { at } q=\frac{2 a b-(a+b) p}{a+b-2 p} .
\end{gathered}
$$

The holographic EOP is computed from the length of the entanglement wedge cross section $\Sigma_{A B}^{\min }$ in the hyperbolic space $d s^{2}=\left(d x^{2}+d z^{2}\right) / z^{2}$, which is a time slice of Poincare $\mathrm{AdS}_{3}$. The geodesic curve $\Sigma_{A B}^{\min }$ is a part of a circle which connects $P:(x, z)=(p, \epsilon)$ and $Q:(x, z)=$ $(q, \epsilon)$, as depicted in Fig. 2. The minimal length condition requires that $\Sigma_{A B}^{\min }$ is perpendicular to the circle $[x-(a+b / 2)]^{2}+z^{2}=\left[(b-a)^{2} / 4\right]$ at their intersection point $(x, z)=\left(x_{0}, z_{0}\right)$. This fixes the value of $q$ as Eq. (14) and we obtain $x_{0}=[p q-a b / p+q-(a+b)]$ and $z_{0}=$ $\sqrt{\left[(b-a)^{2} / 4\right]-\left[x_{0}-(a+b / 2)\right]^{2}}$. Its length $A\left(\Sigma_{A B}^{\min }\right)$ is computed as

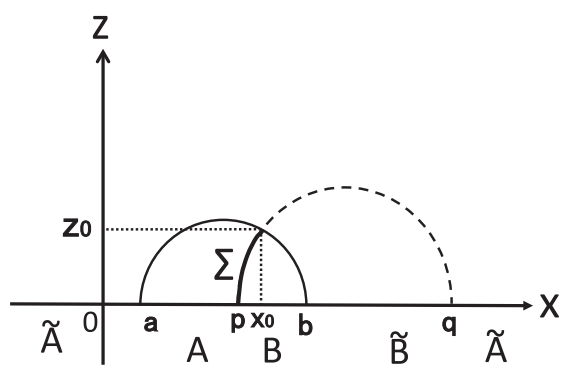

FIG. 2. Calculation of Holographic EOP. The length of $\Sigma$ (thick curve in the above picture) multiplied by $\left(1 / 4 G_{N}\right)=(c / 6)$ gives the holographic EOP, Eq. (3).

$$
\frac{q-p}{2} \int_{\epsilon}^{z_{0}} \frac{d z}{z \sqrt{\frac{(q-p)^{2}}{4}-z^{2}}}=\log \left[\frac{2(p-a)(p-b)}{\epsilon(b-a)}\right] .
$$

Thus the holographic EOP Eq. (3) perfectly matches with Eq. (13).

It is straightforward to extend the above agreement between $E_{W}\left(\rho_{A B}\right)$ and $S_{A \tilde{A}}$ to the finite temperature case by performing a conformal transformation $y=e^{(2 \pi / \beta) \zeta}$. Similarly, we can extend it to the finite size case.

Optimization of double intervals.-As the second class of examples, we consider the case where the subsystems $A$ and $B$ are mutually disconnected intervals on an infinite line. Though we focus on the vacuum state in a two-dimensional CFT below, it is straightforward to extend to the finite temperature setup by the conformal map $y=e^{(2 \pi / \beta) \zeta}$ as before.

The reduced density matrix $\rho_{A B}$ is described by a Euclidean path integral on the complex plane ( $y$ plane) with two slits along $A$ and $B$ as in the upper left picture of Fig. 3. Since $\rho_{A B}$ depends only on the cross ratio $\eta$ owing to the conformal invariance, without losing generality, we can fully parametrize the subsystem $A$ and $B$ as

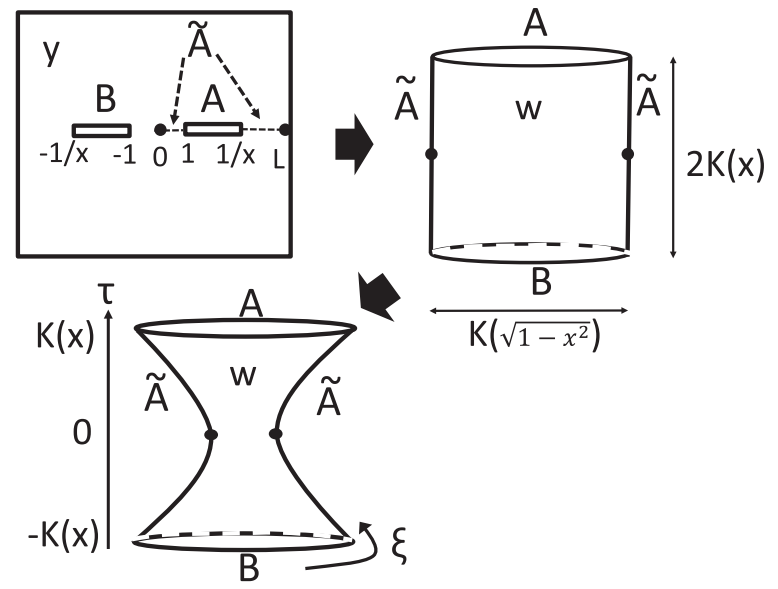

FIG. 3. Conformal map from complex plane with two cuts to a cylinder and its path-integral optimization. 


$$
A=[1,1 / x], \quad B=[-1 / x,-1], \quad(0<x<1) .
$$

The cross ratio is given by $\eta=(1-x)^{2} / 4 x$. We can choose the subsystem $\tilde{A}$ and $\tilde{B}$ which purify $\rho_{A B}$ such that $A \tilde{A}=$ $[0, L]$ and take the limit $L \rightarrow \infty$, owing to the reflection symmetry. We confirmed numerically that this reflection symmetry is not spontaneously broken.

The $y$ plane with two slits is mapped into a cylinder, which is described by the coordinate $w=\xi+i \tau$, as

$$
w=i \int_{0}^{y} d \tilde{y} \frac{1}{\sqrt{\left(1-\tilde{y}^{2}\right)\left(1-x^{2} \tilde{y}^{2}\right)}},
$$

as in the upper right of Fig. 3.

Finally, we perform the path-integral optimization on a cylinder as in the lower picture of Fig. 3. We obtain the optimized metric by solving the Liouville equation:

$$
d s^{2}=e^{2 \phi} d w d \bar{w}=e^{2 \tilde{\phi}} d y d \bar{y}
$$

where

$$
\begin{aligned}
e^{2 \phi} & =\frac{\pi^{2}}{4 K(x)^{2}} \frac{\epsilon^{2}}{\cos ^{2}\left(\frac{\pi \tau}{2 K(x)}\right)}, \\
e^{2 \tilde{\phi}} & =e^{2 \phi} \frac{1}{\left|\left(1-y^{2}\right)\left(1-x^{2} y^{2}\right)\right|^{2}} .
\end{aligned}
$$

We introduced the elliptic function $K(x)$ :

$$
K(x)=\int_{0}^{1} d \tilde{y} \frac{1}{\sqrt{\left(1-\tilde{y}^{2}\right)\left(1-x^{2} \tilde{y}^{2}\right)}} .
$$

The coordinates $\tau$ takes values in the range

$$
-K(x)+\delta_{2} \leq \tau \leq K(x)-\delta_{2},
$$

and $\xi$ is periodically identified: $\xi \sim \xi+2 K\left(\sqrt{1-x^{2}}\right)$. Here $\delta_{2}$ is an infinitesimally small regularization parameter fixed by the condition Eq. (1). Again, the detail of $\delta_{2}$ is not important in our calculations below. The boundary points of $A \tilde{A}, y=0$ and $y=L(\rightarrow \infty)$, are mapped into the antipodal two points in the middle: $(\xi, \tau)=(0,0)$ and $(\xi, \tau)=\left(K\left(\sqrt{1-x^{2}}\right), 0\right)$.

Now the entanglement entropy $S_{A \tilde{A}}^{\min }$ after the optimization can be computed as follows:

$$
\begin{aligned}
S_{A \tilde{A}}^{\min } & =\frac{c}{3} \log \frac{L}{\epsilon}+\left.\frac{c}{6} \tilde{\phi}\right|_{y=L}+\left.\frac{c}{6} \tilde{\phi}\right|_{y=0} \\
& =\frac{c}{3} \log \frac{L}{\epsilon}+\frac{c}{6} \log \left[\frac{\pi \epsilon}{2 L^{2} x K(x)}\right]+\frac{c}{6} \log \left[\frac{\pi \epsilon}{2 K(x)}\right] \\
& =-\frac{c}{6} \log x-\frac{c}{3} \log \left[\frac{2 K(x)}{\pi}\right] .
\end{aligned}
$$

Note that before the optimization, $S_{A \tilde{A}}$ was UV divergent. The optimization procedure performs the Weyl transformation such that it squeezes the metric or equally reduces the number of lattice sites. Accordingly, $S_{A \tilde{A}}$ gets smaller until it becomes finite. As we will argue later, our field theoretic description of this procedure is valid within a certain range.

Since $K(x) \simeq(\pi / 2)+(\pi / 8) x^{2}+\ldots$ for $x \ll 1$, we have

$$
S_{A \tilde{A}}^{\min }=-\frac{c}{6} \log x+O\left(x^{2}\right) .
$$

We can compare this with the holographic EOP $[5,6]$ :

$$
E_{W}\left(\rho_{A B}\right)=-\frac{c}{6} \log x, \quad(0<x<3-2 \sqrt{2}) .
$$

For $3-2 \sqrt{2}<x<1$ we have $E_{W}\left(\rho_{A B}\right)=0$ as the entanglement wedge gets disconnected.

It is also useful to note that the holographic mutual information (HMI) $I(A: B)=S_{A}+S_{B}-S_{A B}$ behaves

$$
\frac{1}{2} I(A: B)=\frac{c}{6} \log z=\frac{c}{6} \log \frac{(1-x)^{2}}{4 x} \quad(0<x<3-2 \sqrt{2}),
$$

while $I(A: B)=0$ for $3-2 \sqrt{2}<x<1$. We plotted these quantities in Fig. 4.

We notice that $S_{A \tilde{A}}^{\min }$ almost coincides with $E_{W}\left(\rho_{A B}\right)$ for $0<x<3-2 \sqrt{2}$ in the plot. However, one may worry that they deviate from each other at the order $O\left(x^{2}\right)$. Also, $S_{A \tilde{A}}^{\min }$ continuously decreases and becomes incorrectly negative as $x$ gets larger. We would like to argue that our Weyl invariance, Eq. (17), breaks down except for $x \ll 1$. This is because the length of minimal cross section of the optimized cylinder, given by $L_{\mathrm{cyl}}=\left[\pi \epsilon K\left(\sqrt{1-x^{2}}\right) / K(x)\right]$, is much greater than the cutoff scale $\epsilon$ only if $x \ll 1$. If the

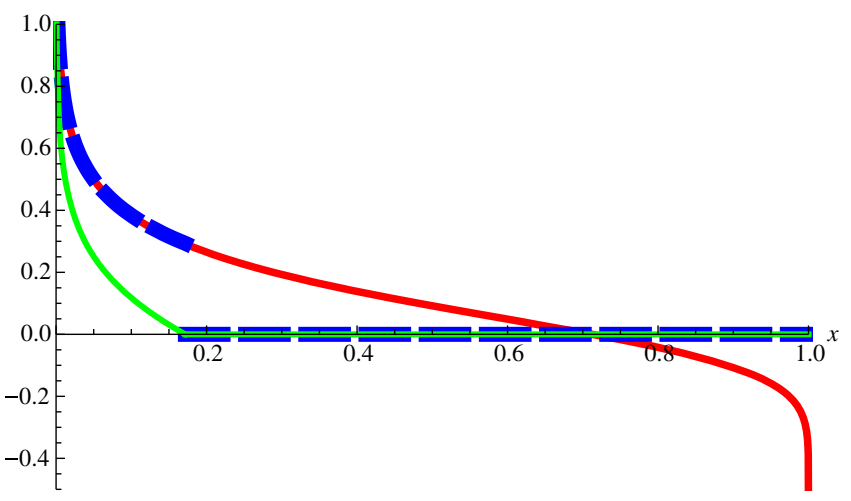

FIG. 4. Plots of HEOP (red), $I(A: B) / 2$ (green), and $S_{A \tilde{A}}^{\min }$ (blue dashed) as a function of $x$. The first two vanish for $x>3-2 \sqrt{2}$. Note that the plot of $S_{A \tilde{A}}^{\min }$ is valid only when $x \ll 1$, where it coincides with HEOP. 
size of the manifold, on which we path integrate, becomes the same order of the lattice spacing $\epsilon$, we cannot trust field theoretic properties of path integrals, such as the Weyl invariance. Therefore, the result, Eq. (21), is trustable only for $x \ll 1$ and in this range the equality $S_{A \tilde{A}}^{\min }=E_{W}\left(\rho_{A B}\right)$ is confirmed. Indeed, in the single interval case, where we found the perfect matching Eq. (13), the Weyl transformation is trustable for any values of $a, b$ and $p$ because we always have $L_{\mathrm{cyl}}=\infty$.

It is also useful to note that at the holographic phase transition point $x=3-2 \sqrt{2}$ (or equally $\eta=1$ ), the minimum cross section of the cylinder becomes $L_{\text {cyl }}=2 \pi \epsilon$. This indeed agrees with the point where a counterpart of confinement or deconfinement transition is expected in the path-integral optimization for holographic CFTs as argued in Ref. [33], though we cannot fully trust this argument because $L_{\text {cyl }}$ gets as small as the lattice spacing $\epsilon$.

Conclusions and discussions. - In this Letter, for a given mixed state $\rho_{A B}$, we introduced a minimized entanglement entropy $S_{A \tilde{A}}^{\min }$ as follows. First we define a special purified state $\left|\Psi_{\min }\right\rangle_{A B C}$ for $\rho_{A B}$ by minimizing the path-integral complexity $C_{L}$ [Eq. (7)]. Then $S_{A \tilde{A}}^{\min }$ is given by taking the minimum of $S_{A \tilde{A}}$ for the purified state $\left|\Psi_{\min }\right\rangle_{A B C}$ among all possible decompositions of the ancilla space for purification $C=\tilde{A} \cup \tilde{B}$.

We analytically computed this quantity $S_{A \tilde{A}}^{\min }$ in several setups in two-dimensional CFTs. Remarkably, we found that it matches with the area of the entanglement wedge cross section $E_{W}\left(\rho_{A B}\right)$ in AdS, called the holographic entanglement of purification.

It is not obvious at present if our special purification is identical to the one which minimizes $S_{A \tilde{A}}$ as in the original definition. Eq. (2). However, we expect they are at least very close to each other because the former minimizes the path-integral complexity and thus efficiently compresses the sizes of $\tilde{A}$ and $\tilde{B}$. In this sense, our result can be regarded as the first quantitative evidence for the holographic EOP conjecture [5,6].

In the original argument [5], the equivalence between $E_{W}\left(\rho_{A B}\right)$ and $E_{P}\left(\rho_{A B}\right)$ was explained by assuming the surface-state duality [40], based on the conjectured relation between tensor networks [41,42] and AdS/CFT [43]. In this argument, the minimization is taken only for quantum states with classical gravity duals. We would like to leave for future works the precise meaning of the minimum in the EOP [Eq. (2)] which correctly matches with $E_{W}\left(\rho_{A B}\right)$. Higher dimensional generalizations are also an intriguing future problem.

We thank Juan Maldacena, Brian Swingle, Kotaro Tamaoka, and Guifre Vidal for useful conversations. M. M. and K. U. are supported by JSPS fellowships. P. C. and T. T. are supported by the Simons Foundation through the "It from Qubit" collaboration. P. C. is supported by JSPS Grant-in-Aid for Research Activity start-up 17H06787. M. M. is supported by JSPS Grant-in-Aid for JSPS Fellows No. 16J08909. T. T. is supported by JSPS Grant-in-Aid for Scientific Research (A) No. 16H02182 and by JSPS Grant-in-Aid for Challenging Research (Exploratory) 18K18766. T. T. is also supported by World Premier International Research Center Initiative (WPI Initiative) from the Japan Ministry of Education, Culture, Sports, Science and Technology (MEXT). K. U. is supported by JSPS Grant-in-Aid for JSPS Fellows No. 18J2288 8.

[1] J. M. Maldacena, The Large N limit of superconformal field theories and supergravity, Int. J. Theor. Phys. 38, 1113 (1999); Adv. Theor. Math. Phys. 2, 231 (1998).

[2] S. Ryu and T. Takayanagi, Holographic Derivation of Entanglement Entropy from AdS/CFT, Phys. Rev. Lett. 96, 181602 (2006); Aspects of holographic entanglement entropy, J. High Energy Phys. 08 (2006) 045.

[3] V.E. Hubeny, M. Rangamani, and T. Takayanagi, A covariant holographic entanglement entropy proposal, J. High Energy Phys. 07 (2007) 062.

[4] B. M. Terhal, M. Horodecki, D. W. Leung, and D. P. DiVincenzo, The entanglement of purification, J. Math. Phys. 43, 4286 (2002).

[5] K. Umemoto and T. Takayanagi, Entanglement of purification through holographic duality, Nat. Phys. 14, 573 (2018).

[6] P. Nguyen, T. Devakul, M. G. Halbasch, M. P. Zaletel, and B. Swingle, Entanglement of purification: From spin chains to holography, J. High Energy Phys. 01 (2018) 098.

[7] K. Umemoto and Y. Zhou, Entanglement of purification for multipartite states and its holographic dual, J. High Energy Phys. 10 (2018) 152.

[8] B. Czech, J. L. Karczmarek, F. Nogueira, and M. Van Raamsdonk, The gravity dual of a density matrix, Classical Quantum Gravity 29, 155009 (2012).

[9] A. C. Wall, Maximin surfaces, and the strong subadditivity of the covariant holographic entanglement entropy, Classical Quantum Gravity 31, 225007 (2014).

[10] M. Headrick, V. E. Hubeny, A. Lawrence, and M. Rangamani, Causality and holographic entanglement entropy, J. High Energy Phys. 12 (2014) 162.

[11] M. Freedman and M. Headrick, Bit threads and holographic entanglement, Commun. Math. Phys. 352, 407 (2017).

[12] V. E. Hubeny, Bulk locality and cooperative flows, J. High Energy Phys. 12 (2018) 068.

[13] S. X. Cui, P. Hayden, T. He, M. Headrick, B. Stoica, and M. Walter, Bit threads and holographic monogamy, arXiv: 1808.05234.

[14] C. A. Agon, J. de Boer, and J. F. Pedraza, Geometric aspects of holographic bit threads, arXiv:1811.08879.

[15] J. Hauschild, E. Leviatan, J. H. Bardarson, E. Altman, M. P. Zaletel, and F. Pollmann, Finding purifications with minimal entanglement, Phys. Rev. B 98, 235163 (2018).

[16] A. Bhattacharyya, T. Takayanagi, and K. Umemoto, Entanglement of purification in free scalar field theories, J. High Energy Phys. 04 (2018) 132. 
[17] H. Hirai, K. Tamaoka, and T. Yokoya, Towards entanglement of purification for conformal field theories, Prog. Theor. Exp. Phys. (2018) 063B03.

[18] J. Kudler-Flam and S. Ryu, Entanglement negativity and minimal entanglement wedge cross sections in holographic theories, arXiv:1808.00446.

[19] K. Tamaoka, Entanglement wedge cross section from the dual density matrix, arXiv:1809.09109.

[20] N. Bao and I. F. Halpern, Holographic inequalities and entanglement of purification, J. High Energy Phys. 03 (2018) 006.

[21] D. Blanco, M. Leston, and G. Perez-Nadal, Gravity from entanglement for boundary subregions, J. High Energy Phys. 18 (2018) 130.

[22] R. Espindola, A. Guijosa, and J. F. Pedraza, Entanglement wedge reconstruction and entanglement of purification, Eur. Phys. J. C 78, 646 (2018).

[23] N. Bao and I. F. Halpern, Conditional and multipartite entanglements of purification and holography, Phys. Rev. D 99, 046010 (2019).

[24] Y. Nomura, P. Rath, and N. Salzetta, Pulling the boundary into the bulk, Phys. Rev. D 98, 026010 (2018).

[25] R. Abt, J. Erdmenger, M. Gerbershagen, C. M. MelbyThompson, and C. Northe, Holographic subregion complexity from kinematic space, J. High Energy Phys. 01 (2019) 012.

[26] A. May and E. Hijano, The holographic entropy zoo, J. High Energy Phys. 10 (2018) 036.

[27] Y. Chen, X. Dong, A. Lewkowycz, and X. L. Qi, Modular flow as a disentangler, J. High Energy Phys. 12 (2018) 083.

[28] E. Caceres and M. L. Xiao, Complexity-action of singular subregions, arXiv:1809.09356.

[29] J. C. Cresswell, I. T. Jardine, and A. W. Peet, Holographic relations for OPE blocks in excited states, arXiv:1809 .09107.

[30] R. Q. Yang, C. Y. Zhang, and W. M. Li, Holographic entanglement of purification for thermofield double states and thermal quench, J. High Energy Phys. 01 (2019) 114.

[31] N. Bao, A. Chatwin-Davies, and G. N. Remmen, Entanglement of purification and multiboundary wormhole geometries, J. High Energy Phys. 02 (2019) 110.
[32] N. Bao, Minimal purifications, wormhole geometries, and the complexity $=$ action proposal, arXiv:1811.03113.

[33] P. Caputa, N. Kundu, M. Miyaji, T. Takayanagi, and K. Watanabe, Anti-de Sitter Space from Optimization of Path Integrals in Conformal Field Theories, Phys. Rev. Lett. 119, 071602 (2017); Liouville action as path-integral complexity: From continuous tensor networks to AdS/CFT, J. High Energy Phys. 11 (2017) 097.

[34] B. Czech, Einstein Equations from Varying Complexity, Phys. Rev. Lett. 120, 031601 (2018).

[35] A. Bhattacharyya, P. Caputa, S. R. Das, N. Kundu, M. Miyaji, and T. Takayanagi, Path-integral complexity for perturbed CFTs, J. High Energy Phys. 07 (2018) 086.

[36] A. M. Polyakov, Quantum geometry of bosonic strings, Phys. Lett. 103B, 207 (1981).

[37] D. Stanford and L. Susskind, Complexity and shock wave geometries, Phys. Rev. D 90, 126007 (2014); A. R. Brown, D. A. Roberts, L. Susskind, B. Swingle, and Y. Zhao, Holographic Complexity Equals Bulk Action, Phys. Rev. Lett. 116, 191301 (2016); L. Lehner, R. C. Myers, E. Poisson, and R. D. Sorkin, Gravitational action with null boundaries, Phys. Rev. D 94, 084046 (2016).

[38] T. Takayanagi, Holographic spacetimes as quantum circuits of path-integrations, J. High Energy Phys. 12 (2018) 048.

[39] C. Holzhey, F. Larsen, and F. Wilczek, Geometric and renormalized entropy in conformal field theory, Nucl. Phys. B424, 443 (1994).

[40] M. Miyaji and T. Takayanagi, Surface/state correspondence as a generalized holography, Prog. Theor. Exp. Phys. (2015) $073 \mathrm{~B} 03$.

[41] G. Vidal, A Class of Quantum Many-Body States that can be Efficiently Simulated, Phys. Rev. Lett. 101, 110501 (2008); Entanglement Renormalization, Phys. Rev. Lett. 99, 220405 (2007).

[42] G. Evenbly and G. Vidal, Tensor Network Renormalization, Phys. Rev. Lett. 115, 180405 (2015); Tensor Network Renormalization Yields the Multi-Scale Entanglement Renormalization Ansatz, Phys. Rev. Lett. 115, 200401 (2015).

[43] B. Swingle, Entanglement renormalization and holography, Phys. Rev. D 86, 065007 (2012). 